\title{
ANTAGONISTIC DRIVE MECHANISM TO INCREASE IMPULSIVE FORCE INSPIRED BY EXOSKELETON SPRING SYSTEM OF MANTIS SHRIMP
}

\author{
SHUNICHI KURUMAYA*, FUMIO ITO* \\ *Department of Precision Mechanical Engineering, Chuo University, 1-13-27, Kasuga, Bunkyo-ku, \\ Tokyo, Japan \\ E-mail: s_kurumaya@bio.mech.chuo-u.ac.jp, f_ito@bio.mech.chuo-u.ac.jp, \\ r_ono@bio.mech.chuo-u.ac.jp,nakamura@mech.chuo-u.ac.jp \\ https://www.chuo-u.ac.jp \\ RIKI ONO*, KATUSHI KAGAYA** and TARO NAKAMURA* \\ ** Next Generation Artificial Intelligence Research Center, Graduate School of Information Science and \\ Technology, The University of Tokyo, 113-0033, Nagase-hongo building 8F, 5-24-5, Hongo, Bunkyo-ku, \\ Tokyo, Japan \\ E-mail:kkagaya@g.ecc.u-tokyo.ac.jp
}

\begin{abstract}
In this paper, a mechanism for generating an impulsive force by using the distortion of an exoskeleton is described. Pneumatic artificial muscles not only have a high power to weight ratio, but also have a high affinity with the human body due to their use of air pressure. However, pneumatic artificial muscles have a slow stretch response rate due to their viscous properties. Therefore, earthworm type mobile robots and wearable assistive devices using artificial muscles move slowly and have difficulty assisting human movements. We focus on the mechanism of force generation using the distortion of the exoskeleton, similar to what is seen in the punching of a mantis or the jumping of a grasshopper, as a countermeasure against the delay in the response rate of the artificial muscle. In this study, we propose a new mechanism to generate a shooting force by incorporating an element similar to an exoskeleton spring into the antagonistic drive mechanism of an artificial muscle. We designed and developed a prototype then operated it and measured the impulsive force. It was found that the developed mechanism showed similar joint movements through muscle contraction to those of mantis shrimp and grasshoppers. Furthermore, it was shown that the time constant for the maximum strike force was $6.6 \mathrm{~N}$ and $63.2 \%$ of the maximum impulsive force was $0.183 \mathrm{~s}$.
\end{abstract}

\section{Introduction}

Pneumatic artificial muscles not only have the advantage of high affinity with the human body due to compliance and shape flexibility derived from air pressure, but also have a high output mass ratio $[1,2]$. Because of these characteristics, pneumatic artificial muscles are used in mobile robots $[3,4]$ and wearable movement assistive device [5,6].

One of the problems with pneumatic artificial muscles is that their contraction speed is too slow for practical use [4]. When pneumatic artificial muscles are used as actuators for earthworm type mobile robots, they cause the robots to run under high payload with their high power to weight ratio. When pneumatic artificial muscles are used in wearable movement assistive devices, it is difficult for the muscles to maintain the human movement speed and provide sufficient assistance [5]. The slowness of the muscles is caused by the artificial muscle's viscosity [7].

Several measures have been taken to solve the problem of movement speed. Two methods of improving the movement speed of earthworm robots are 1) varying both pneumatic-airpressure and time [8], and 2) increasing the artificial muscle's amount of contraction [9]. In addition, feed-forward control can be combined with these methods to accomplish a natural 
assist by letting human movement follow the movement of the artificial muscle. Although these methods improve the speed of the contractive response of the artificial muscle, they are not practical solutions. In addition, the speed of the contraction response of these methods is being improved.

Therefore, we focused on the impact generation mechanism that uses the distortion of the exoskeleton, such as the punch of a mantis shrimp or the jump of a grasshopper and applied it to the musculoskeletal drive system to solve the problem of slow contraction response. Mantis shrimp and grasshoppers compensate the slow response of living muscles by combining exoskeleton spring elements and realizing a large instantaneous force [10, 11, 12]. A mantis shrimp's punch has an interesting ability to cause cavitation from its speed; thus, the mantis shrimp is studied as a biomimetic robot for cavitation generation. Living organisms in the natural world use the contraction of living muscles as actuators to perform various movements $[13,14]$. When focusing on skeletal structure, organisms are classified into two types: 1) endoskeleton, found in humans and large organisms and 2) exoskeleton, found in arthropods such as insects and crustaceans. The endoskeleton is a structure where muscles are attached around the skeleton and is excellent for skeletal stability, motility, and large power output due to muscle hypertrophy. Therefore, the endoskeleton is commonly used for driving biomimetic robots $[15,16]$. In contrast, the exoskeleton has a structure where muscles are inside the skeleton and is used in tendon driven robots that are strong against the surrounding environment and external enemies [17]. A mantis shrimp, as a preparatory action for punching, distorts the exoskeleton system in the appendage to store elastic energy. When punching, the flexor muscle is relaxed, and a large force is instantaneously generated almost by the elastic force of the exoskeleton. Similarly, an exoskeleton spring called the semi-lunar spring at the rear leg knee joint of the grasshopper contributes to an instantaneous output of a large force. In this study, we aim to overcome the instantaneous power of the artificial muscle pneumatic drive system by imitating the exoskeleton spring mechanism; the spring mechanism has not been used in exoskeleton-based biomimetic robots with artificial muscle and this instantaneous power has been difficult to obtain.

In this study, we propose an impact generation mechanism that incorporates an element similar to the exoskeleton spring into the antagonistic drive mechanism of the artificial muscle. This model mimics the joint mechanism of a mantis shrimp or grasshopper when a shock occurs and is intended for application in mobile robots and human body-mounted motion assist devices. In this paper, we report the design of a prototype that incorporates an exoskeleton spring element into a one-joint antagonistic drive system using a straight-fiber-type artificial muscle [18] and report the experimental results. The axial fiber-reinforced artificial muscle and the mechanism for impact generation using strain of the exoskeleton of the mantis shrimp are described in Sections 2 and 3, respectively. In Section 4, the development of the proposed mechanism and its driving principle are described in addition to an impact generation experiment using the developed mechanism. In Section 5, we summarize this paper and discuss future possibilities.

\section{Straight-Fiber-Type Artificial Muscle}

\subsection{Design}

The straight-fiber-type artificial muscle used in this study is pneumatically driven with a structure where fibers along the axial direction are encompassed in a rubber tube as shown in Figure 1. When air pressure is applied, the structure expands only in the radial direction and contracts in the axial direction due to the constraint of the fibers contained in the artificial muscle. In this study, the artificial muscle applied to the antagonistic driving system has an initial inner diameter of $12 \mathrm{~mm}$ and an initial length of $180 \mathrm{~mm}$. The L / D ratio of the artificial muscle is 
the outer diameter divided by the axial length [19]. Two rings were attached to the artificial muscle to divide it into three equal parts and the $\mathrm{L} / \mathrm{D}$ ratio was set to 5 .

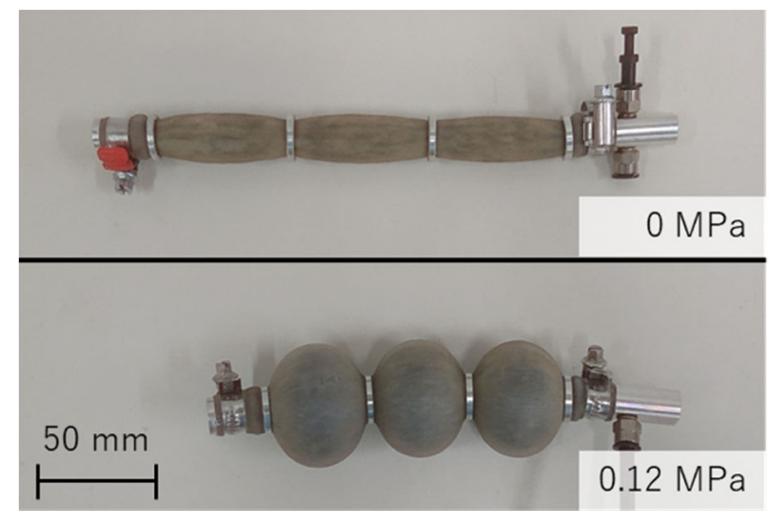

Figure 1. Straight-fiber-type artificial muscle with no air pressure applied (top) and with air pressure of $0.12 \mathrm{MPa}$ applied (bottom).

\subsection{Characteristics}

The air pressure characteristics of the axial fiber reinforced artificial muscle used in this paper can be seen in Figure 2, (a) shows the relationship between applied pressure and the amount of contraction under no load and (b) shows the relationship between applied pressure and the contraction force at the natural length, i.e. the length with no air pressure. The air pressure was raised from $0 \mathrm{MPa}$ to $0.20 \mathrm{MPa}$ in increments of $0.05 \mathrm{MPa}$; and the contraction amount and contraction force were measured. As shown in Figure 2 (a), the amount of contraction monotonically increases with pressure applied to the artificial muscle. In this experiment, the shrinkage in the range of $0 \mathrm{MPa}$ to $0.05 \mathrm{MPa}$ was negligible. This is because the energy in this range is used to extend the crystallization of the natural rubber of the artificial muscle, and the artificial muscle does not expand or contract under low pressure conditions [20]. In the region with low applied pressure, the increase in contraction is considerable, while in the region with high applied pressure, the increase in contraction is gradual. Prior research has demonstrated that, at high pressure, the artificial muscle considerably expands and converges to a shape constrained by the fiber; the amount of contraction then converges to a constant value [7].

As shown in Figure 2 (b), the contraction force is proportional to the applied pressure, with a slope of $2.5 \times 10^{3} \mathrm{~N} / \mathrm{MPa}$. In contrast, no contraction force was generated when low pressure was applied. This is also due to the extension of the crystallization of the natural rubber.

(a)

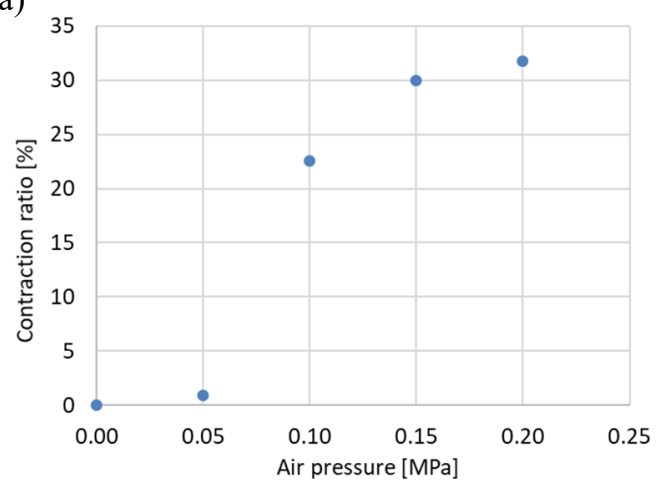

(b)

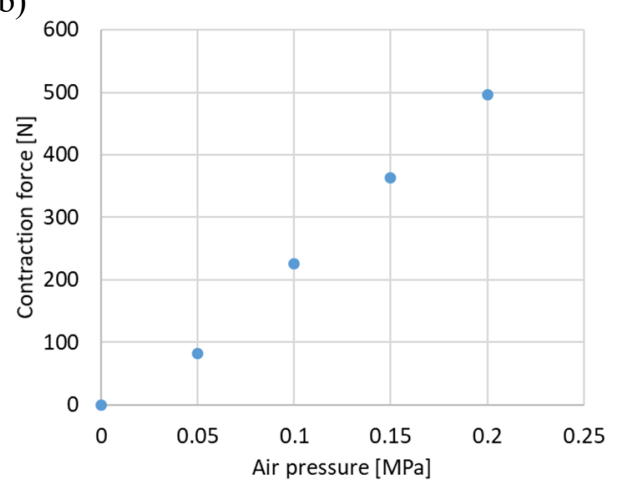

Figure 2. Air pressure characteristics of the straight-fiber-type artificial muscle. Relationship between (a) applied pressure and contraction ratio under no load, and (b) applied pressure and contraction force. 


\section{Antagonistic Drive Mechanism with Exoskeleton Spring}

\subsection{Design}

In this paper, we design an antagonistic drive mechanism that incorporates an element of the exoskeleton spring similar to that of the mantis shrimp's raptorial appendage. The appendage of the mantis shrimp is composed of merus, carpus, propodus and dactyl. Carpus, propodus and dactyl are united to form a strike body when the shrimp performs strike. Prior to the initiation of the strike, the dorsal saddle and the ventral bar mainly deform and function as a spring system that stores elastic energy. The flexor and extensor muscles in the merus co-contract to prevent the strike body from the movement. Thus, the process of mantis shrimp's punch can be divided into two phases: the preparatory loading and unloading strike movement, as shown below.

\subsubsection{Loading for preparation (mantis shrimp)}

Flexing the joint between merus and carpus by contracting flexor muscles (Figure 3 (a)). At this time, the state is fixed by the tendon of the flexor muscle and the latch mechanism in the exoskeleton. When the co-contraction occurs, the exoskeleton deforms and it stores elastic energy (Figure $3(\mathrm{~b})$ ).

\subsubsection{Unloading for strike (mantis shrimp)}

Relaxing the flexor muscles releases the latch mechanism and elastic energy stored in the exoskeleton. The joint is extended by the combination of the tensile force of the extensor and the elastic force of the exoskeleton. The strike bodyis then pushed out.
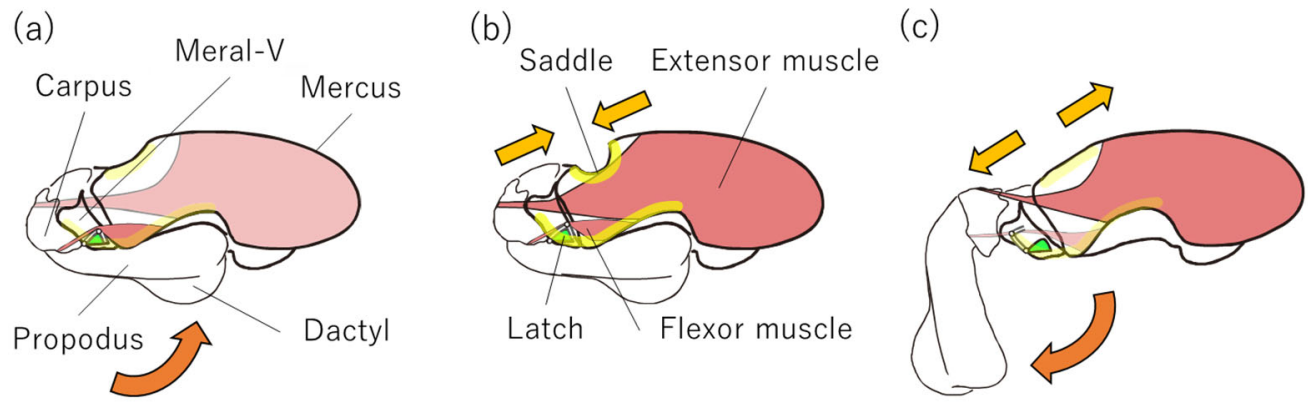

Figure 3. The flow of the punch motion by the mantis shrimp's raptorial limb. (a) latch fixation and flexion by contraction of flexor muscles. (b) deformation of exoskeleton spring and increase of elastic energy due to the co-contraction of extensor and flexor muscle. (c) release and extension of the latch by relaxation of flexor muscles.

\subsection{Development}

We developed a mechanism to increase the impact force by incorporating the saddle element, the exoskeleton spring described in the previous section, into the antagonistic drive system. The developed one-joint antagonistic drive mechanism (Figure 4) an axial fiber-reinforced artificial muscle attached as a flexion / extension muscle. The exoskeleton spring was installed so that the tension spring strengthens the moment of the extensor to antagonize the flexor. The mechanism that the exoskeleton distorts when the rotation axis moves parallel to the artificial muscle is reproduced. 


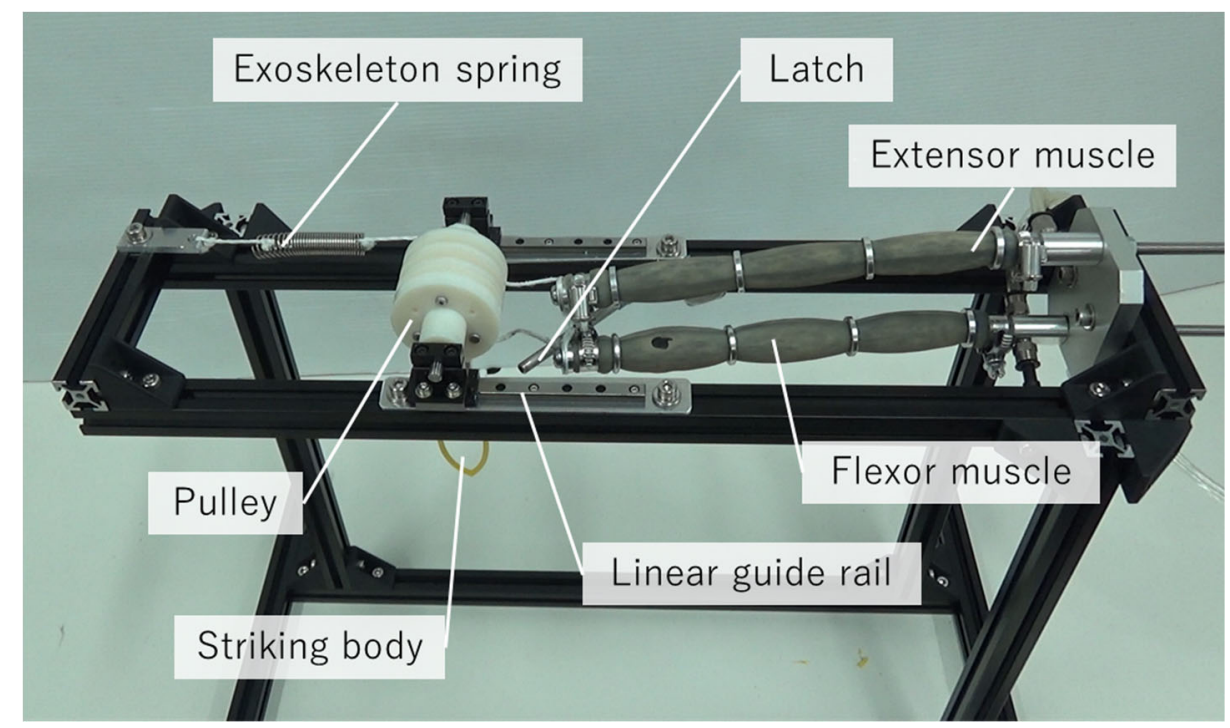

Figure 4. Prototype of one-joint antagonist driving mechanism inspired by exoskeleton spring.

\subsection{Experiment}

Two experiments, a motion experiment and hitting experiment, were performed using a onejoint antagonistic driving mechanism inspired by the developed exoskeleton spring system. From the initial state (Figure 5 (a)), the bending motion was performed by applying a pneumatic pressure of $0.20 \mathrm{MPa}$ to the flexor muscle (Figure 5 (b)), and the rotation center was moved by applying a pneumatic pressure of $0.20 \mathrm{MPa}$ to the extensor muscle; elastic energy was then stored in the spring (Figure 5 (c)). The preparatory loading operation was confirmed. The joint was extended by applying no air pressure to the flexor muscle from the preparatory loading state and the unloading motion, which is similar to a mantis shrimp's punch, was confirmed (Figure $5(d))$.

\subsubsection{Loading for preparation (our system)}

Pneumatic pressure was applied to the flexor muscles to contract and flex the joints. At this time, the rotating shaft did not move because the tensile force of the bending muscle and the tension spring were balanced. Then, by applying air pressure to the extensor muscle for cocontraction, the center of rotation moved parallel to the artificial muscles. At this time, the joint remained bent.

\subsubsection{Unloading for strike (our system)}

By stopping the application of air pressure to the flexor muscle and evacuating the air, the flexor muscle relaxed and the joint was stretched by the tensile force of the extensor muscle and spring.

Next, the impulsive force of the developed mechanism was measured. Figure 6 shows the setup of the experimental method. An aluminum arm was attached to the antagonistic joint and the plate on which the load cell was attached was designed to hit vertically when the arm rotated. The impact force at this instant was read by a load cell and measured using a logger (d-Space) with a sampling time of $\sim 20 \mathrm{kHz}$. The air pressure application time of the artificial muscle was controlled by a solenoid valve (CKD 3-6-2-B) with an Arduino Uno. For the preliminary operation, air pressure is applied to the flexor muscle for $1.5 \mathrm{~s}$. Then, air pressure was applied to the extensor muscle for $1.5 \mathrm{~s}$ as well. For the launching operation, the air pressure to the flexor muscle was exhausted to generate a punch. $0.5 \mathrm{~s}$ after, the air pressure to the extensor muscle 
was the air was exhausted. The applied pressure to each artificial muscle was constant, 0.20 MPa.

From the experimental results, it was confirmed that the maximum impact force of this mechanism was $6.6 \mathrm{~N}$, and the time constant at which it was $63.2 \%$ of the maximum impact force was $77.7 \mathrm{~ms}$ (Figure 7). In this section, the movement and impact force were confirmed by imitating a mantis shrimp's punch using a one-joint antagonistic drive mechanism inspired by exoskeleton springs.

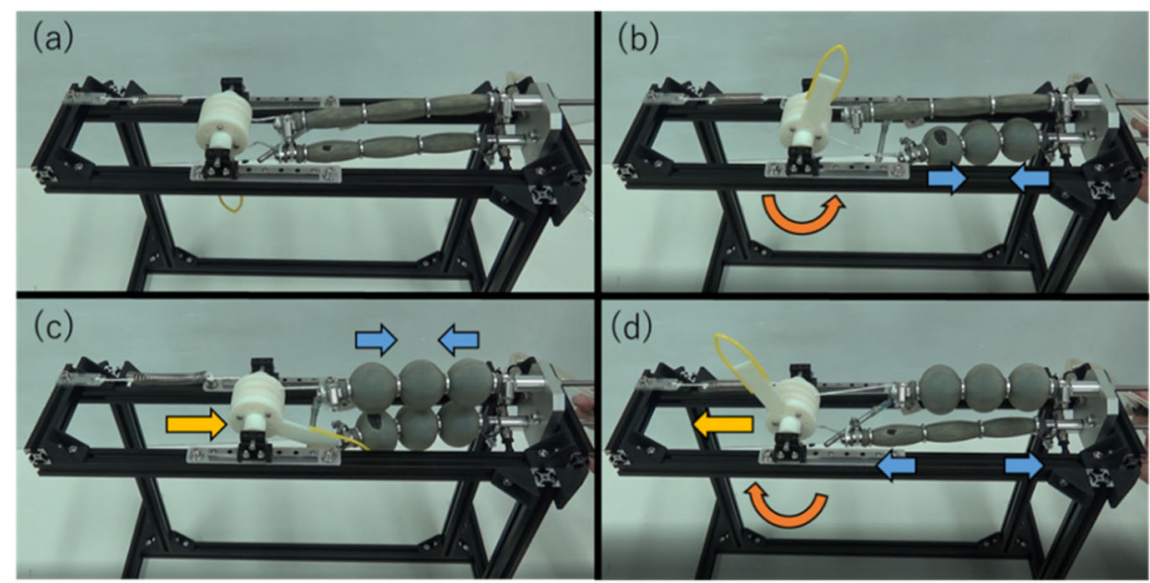

Figure 5. The steps of the antagonistic drive mechanism experiment inspired by the exoskeleton spring. (a) initial state. (b) latch fixation and flexion by contraction of flexor muscles. (c) translation of the rotation axis and the increased elastic energy of the exoskeleton by contraction of the extensor and flexor muscles. (d) release and extension of the latch by relaxation of flexor muscles

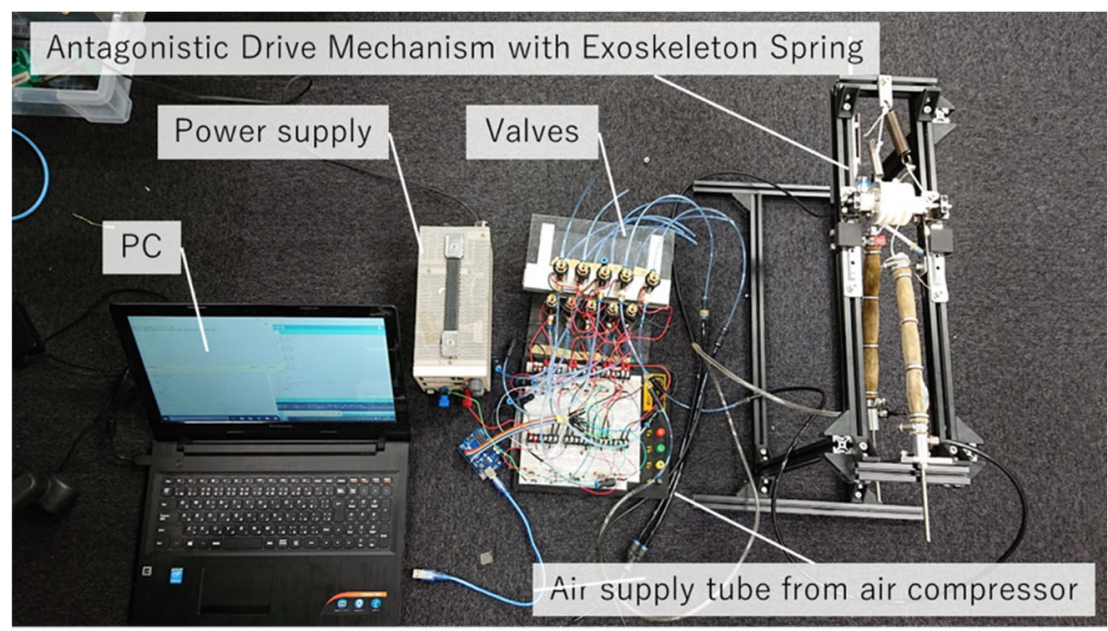

Figure 6. Experimental setup of the antagonistic drive mechanism inspired by the exoskeleton spring. 


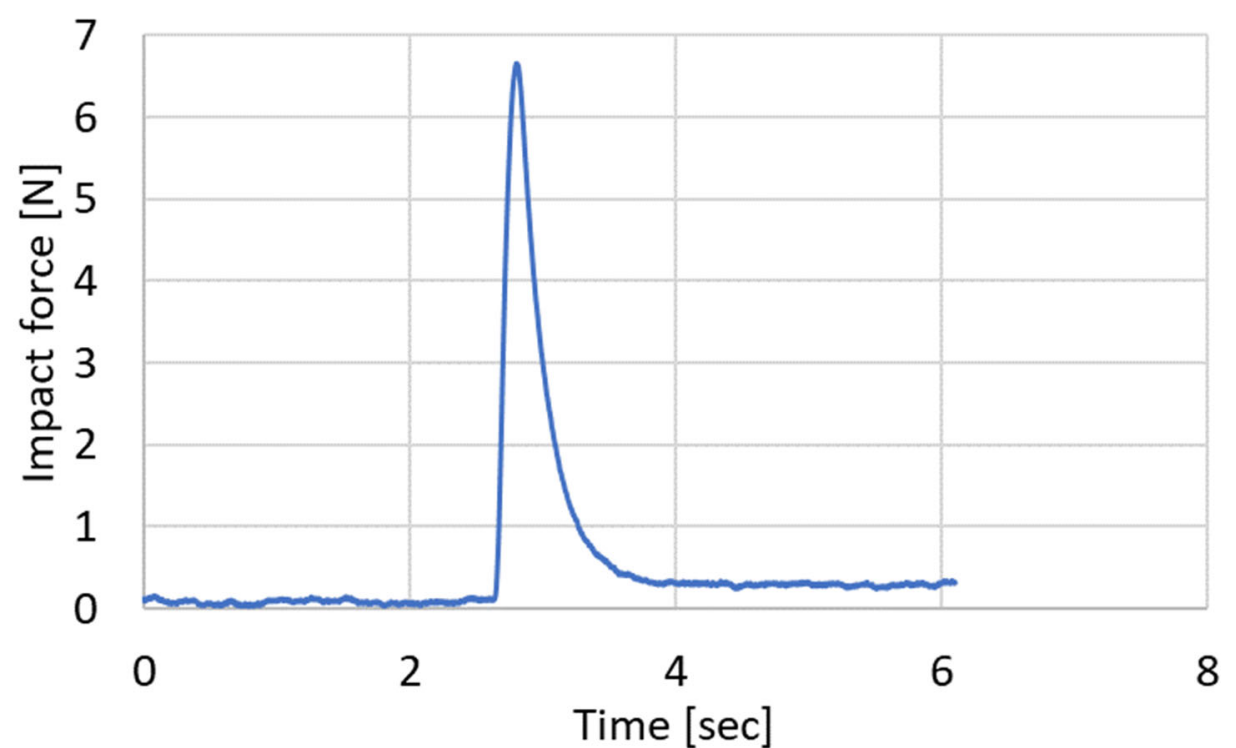

Figure 7. The result of a shock force measurement experiment of an antagonistic drive mechanism. The sampling time is $20 \mathrm{kHz}$.

\section{Conclusions}

In this study, we focused on the exoskeleton springs found in mantis shrimps and grasshoppers. We designed and developed a one-joint antagonistic driving mechanism prototype inspired by the exoskeleton springs of the mantis shrimp and performed operation and impact measurement experiments. The mechanism developed from the experimental results showed a joint movement by muscle contraction similar to that of the mantis shrimp and grasshopper. The impact test showed that the maximum impact force of this mechanism was $6.6 \mathrm{~N}$, and the time constant for $63.2 \%$ of the maximum impact force was $77.7 \mathrm{~ms}$. In the future, the mechanical characteristics of the prototype mechanism will be evaluated to clarify the effect of exoskeleton spring elements on antagonistic driving.

\section{Acknowledgments}

The part of this research was supported by JSPS KAKENHI Grant Numbers 19H05334.

\section{References}

1. F. Daerden, D. Lefeber, B. Verrelst, and R. V. Ham, "Pleated pneumatic artificial muscles: compliant robotic actuators," in Proc. IEEE Int. Conf. on Intell. Robots Syst., Mad, Hawaii, 2001, pp. 1958-1963.

2. S. Kurumaya, H. Nabae, G. Endo, and K. Suzumori, "Design of thin McKibben muscle and multifilament structure," Sens. Actuators A. Phys., vol. 261, pp. 66-74, 2017.

3. H. Tsukagoshi, K. Terashima, and Y. Takai, "A self-propelled catheter capable of generating travelling waves with steering function by mono-line drive," in Proc. IEEE Int. Conf. Robot. Autom. (ICRA).

4. N. Saga, T. Nakamura, and S. Ueda, "Study on peristaltic crawling robot using artificial muscle actuator," in Proc. IEEE/ASME Int. Conf. Adv. Intell. Mechatron, 2003, pp. 679684.

5. R. Suzuki, M. Okui, S. Iikawa, Y. Yamada, and T. Nakamura, "Novel feedforward controller for straight-fiber-type artificial muscle based on an experimental identification 
model," in Proc. First IEEE-RAS Int. Conf. Soft Robot. (RoboSoft), Livorno, Italy, WeBT.3, Apr. 2018, pp. 31-38.

6. S. Koizumi, et al., "Soft robotic gloves with thin McKibben muscles for hand assist and rehabilitation," in 2020 IEEE/SICE Int. Symp. Syst. Integr. (SII), Honolulu, HI, USA, 2020, pp. 93-98.

7. A. Kojima, M. Okui, I. Hisamichi, T. Tsuji, and T. Nakamura, "Straight-fiber-type artificial muscle deformation under pressurization," IEEE Robot. Autom. Lett., Feb. 2019. DOI [10.1109/LRA2019.2902016]

8. T. Tomita, T. Tanaka, and T. Nakamura, "Development of a peristaltic crawling robot for long-distance sewer pipe inspection with consideration of complex pipe line," in Proc. IEEE Int. Conf. Intell. Robots Syst., Hamburg, Germany, (IROS 2015) Oct. 2015, pp. 2742-2747.

9. F. Ito, T. Kawaguchi, M. Kamata, Y. Yamada, and T. Nakamura, "Proposal of a peristaltic motion type duct cleaning robot for traveling in a flexible pipe," in Proc. IEEE/RSJ Int. Conf. Intell. Robot. Syst. (IROS2019), China, Macau, Nov. 2019.

10. S. N. Patek, B. N. Nowroozi, J. E. Baio, R. L. Caldwell, and A. P. Summers, "Linkage mechanics and power amplification of the mantis shrimp's strike," J. Exp. Biol., vol. 210, no. 20, pp. 3677-3688, 2007. doi:10.1242/jeb.006486

11. S. M. Cox, D. Schmidt, Y. Modarres-Sadeghi, and S. N. Patek, "A physical model of the extreme mantis shrimp strike: kinematics and cavitation of Ninjabot," Bioinspiration Biomimetics, vol. 9, no. 1, pp. 016014, Feb. 2014.

12. K. Kagaya, and S. N. Patek, "Feed-forward motor control of ultrafast, ballistic movements," J. Exp. Biol., vol. 219, pp. 319-333, 2016. doi:10.1242/jeb.130518

13. S. N. Patek, and R. L. Caldwell, "Extreme impact and cavitation forces of a biological hammer: strike forces of the peacock mantis shrimp Odontodactylus scyllarus," J. Exp. Biol. vol. 208, no. 19, pp. 3655-3664, 2005. doi:10.1242/jeb.01831

14. X. Tang, and D. Staack, "Bioinspired mechanical device generates plasma in water via cavitation,” Sci. Adv., vol. 5, no. 3, pp. eaau7765, Mar. 2019. DOI: 10.1126/sciadv.aau7765

15. S. Kurumaya, K. Suzumori, H. Nabae, and S. Wakimoto, "Musculoskeletal lower-limb robot driven by multifilament muscles," Robomech J., vol. 3, no. 1, pp. 18, 2016.

16. Y. Asano, K. Okada, and M. Inaba, "Design principles of a human mimetic humanoid: Humanoid platform to study human intelligence and internal body system," Sci. Robot., vol. 2, no. 13, pp. eaaq0899, 2017.

17. S. Kurumaya, H. Nabae, G. Endo, and K. Suzumori. "Exoskeleton inflatable robotic arm with thin Mckibben muscle," in IEEE 1st Int. Conf. Soft Robot., 2018, pp. 120-125.

18. N. Saga, T. Nakamura, et.al, "Development of artificial muscle actuator reinforced by kevlar fiber," in Proc. IEEE Int. Conf. Ind. Technol. (ICIT2002), 2002, pp. 950-954.

19. A. Kojima, M. Okui, Y. Yamada, and T. Nakamura, "Prolonging the lifetime of straightfiber-type pneumatic rubber artificial muscle by shape consideration and material development," in Proc. First IEEE-RAS Int. Conf. Soft Robot. (RoboSoft), Livorno, Italy, WeTT.23, Apr. 2018, pp. 188-195.

20. A. Kojima, M. Okui, I. Hisamichi, N. Tadami, T. Tsuji, and T. Nakamura, "Strain-induced crystallization to prolong the lifetime of pneumatic artificial muscles," in SPIE, Smart Struct. Nondestruct. Eval., Denver, Colorado, USA, Mar. 2019, pp. 109650X. 